\title{
TITLE:
}

\section{Close-loop angle control of stepper motor fed by power packets}

$\operatorname{AUTHOR}(S)$ :

Mochiyama, Shiu; Takahashi, Ryo; Hikihara, Takashi

\section{CITATION:}

Mochiyama, Shiu ... [et al]. Close-loop angle control of stepper motor fed by power packets. IEICE Transactions on Fundamentals of Electronics, Communications and Computer Sciences 2017, E100A(7): 1571-1574

ISSUE DATE:

2017-07-01

URL:

http://hdl.handle.net/2433/235217

RIGHT:

(C) 2017 IEICE. 


\title{
LETTER \\ Close-Loop Angle Control of Stepper Motor Fed by Power Packets
}

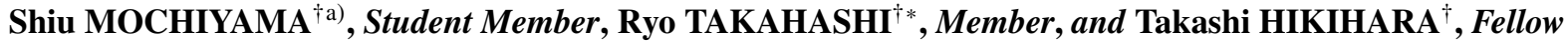

SUMMARY The power packet dispatching system, in which electric power is transferred in a pulse-shaped form with information, is expected to realize dynamical management of multiple power sources in independent systems such as robots. In this letter, close-loop control of a stepper motor by power packets is discussed. The precise angle control is achieved by the combined transfer of power and control information in experiments.

key words: power packet, stepper motor, close-loop control, angle control

\section{Introduction}

A wide variety of systems, including a legged robot [1], an electric vehicle [2], and a More Electric Aircraft [3], are driven by electricity. Recently dispersed power sources, such as photovoltaic cells, fuel cells, and batteries, have been introduced to these systems. Aiming for efficient management of these multiple power sources, a power packet dispatching system was proposed [4]. A power packet is a pair of pulse-shaped dc power and information tags attached in voltage waveforms. In a dispatching network, power packets are transferred to their destination loads according to the address information in the tags. The authors' group has already developed prototype apparatuses to produce and forward power packets, and verified their experimental feasibility [4], [5]. The power packet dispatching based on a physical tag attachment can realize a simultaneous handshake of energy and information in power management.

This study focuses on an application of the power packet dispatching to an electrically independent system, which is disconnected from external power sources. The research goal is to realize an optimal power distribution under the limits of power available and demand of loads. We seek the distributed and autonomous control of the flow of power packets. This kind of decentralization is better suited for a network including many elements; centralized control restricts the performance and throughput as the number of elements increases. For this purpose, we need to ensure the simultaneity of power and information transfer. In conventional systems, power supply system and information system including control system are designed independently under the assumption that their power sources

\footnotetext{
Manuscript received January 27, 2017.

Manuscript revised April 3, 2017.

${ }^{\dagger}$ The authors are with the Department of Electrical Engineering, Kyoto University, Kyoto-shi, 615-8510 Japan.

*Presently, with Aichi University of Technology.

a)E-mail: s-mochiyama@dove.kuee.kyoto-u.ac.jp

DOI: 10.1587/transfun.E100.A.1571
}

are large enough to feed every load. After power demands are calculated for individual loads, the requested power is supplied by current regulation via converters just in front of loads. However, since independent systems contain only a limited amount of power, the assumption of large source capacity does not always hold. A simple adoption of the conventional system may cause an imbalance of calculated demand and actual power flow; in the worst case, it leads to maloperation or damage of loads. Power packet dispatching system can solve the problem by the physical integration of power transfer and related information.

Toward the application of the power packet dispatching to independent systems, this letter discusses the feasibility of motion control by power packets. So far, our group has experimentally verified the feasibility of trajectory control of a manipulator fed by power packets [6]. However, since the study adopts a centralized open-loop control of stepper motors, there remain following two challenges. First, the open-loop scheme can fail to control the angle in the presence of an external disturbance. An angle disturbance can cause a step-out. Second, in [6], the information tag is attached by a centralized scheme at the packet generation, and fixed through the entire transfer from a source to a load. As mentioned above, the centralized control restricts the performance when the whole system gets larger. To overcome these challenges, this letter discusses decentralized closeloop control of stepper motors fed by power packets with the angle feedback. We implement the close-loop scheme to a distributed subsystem for a decentralization of power transfer and control. A part of control information is attached at the packet generation; the rest, or more detailed one, is generated at the distributed subsystem for each power packet with application of sensor feedback information. This setup is to confirm that decentralized motion control can be realized in the power packet dispatching system, which has not been discussed yet in previous studies. The feasibility of the proposed control system is verified in experiments.

Close-loop control of stepper motors has been proposed in some studies, e.g. by Fredriksen [7], which focus on how to generate the control information. On the contrary, we develop a close-loop control scheme in the power packet dispatching system, which successfully integrates the power transfer and control information. 


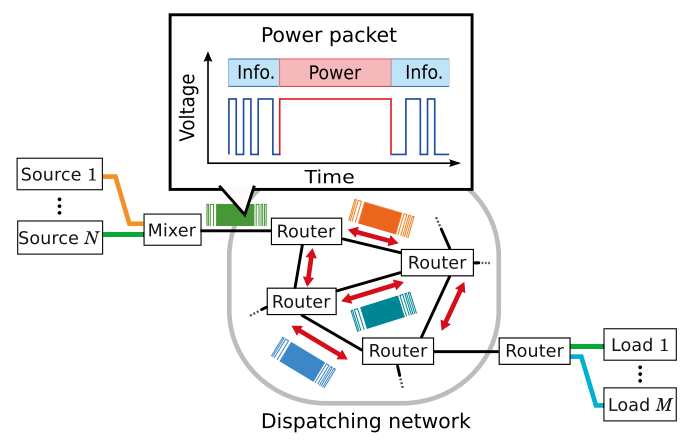

Fig. 1 Power packet and its dispatching system.

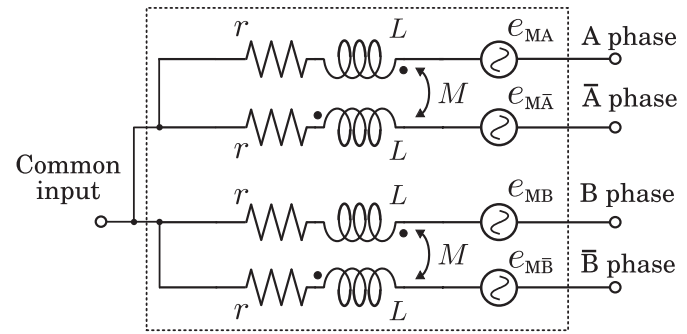

Fig. 2 Electrical circuit expression of stepper motor.

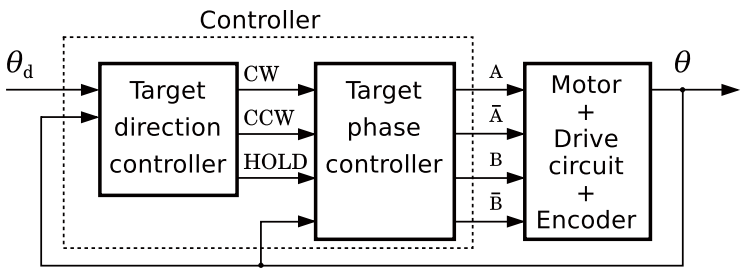

Fig. 3 Flow of information for proposed close-loop motor control.

\section{Close-loop Control Scheme in Power Packet Dis- patching System}

Figure 1 shows a general configuration of the power packet dispatching system. Power packets are generated by a mixer with circuit switching [4]. A power packet consists of pulseshaped power called payload and information tags of voltage logic waveforms. It is forwarded to its destination by routers in the dispatching network according to the tag information [4], [5].

This letter discusses angle control of a unipolar hybrid stepper motor by power packets. This type of motors consist of four excitation phases, A, B, $\bar{A}$ and $\bar{B}$, as shown in Fig. 2 . We adopt a one-phase excitation method [8] for its drive; therefore, the motor angle control is achieved by supplying pulse-shaped power to the phases in an appropriate order.

In this letter, we adopt a close-loop control system as shown in Fig. 3, in reference of [7]. The input and output signals of the controller are target angle $\theta_{\mathrm{d}} \in[0,2 \pi)$ and target excitation phase $p_{\mathrm{d}} \in\{\mathrm{A}, \mathrm{B}, \overline{\mathrm{A}}, \overline{\mathrm{B}}\}$, respectively. The feedback signal of present angle $\theta \in[0,2 \pi)$ is assumed to be available at arbitrary time. By using $\theta_{\mathrm{d}}$ and $\theta$, the controller

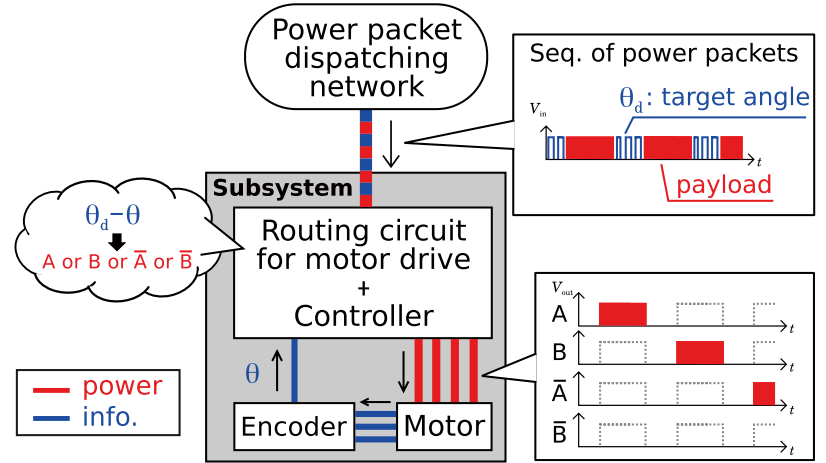

Fig. 4 Schematic of proposed subsystem connected to dispatching network.

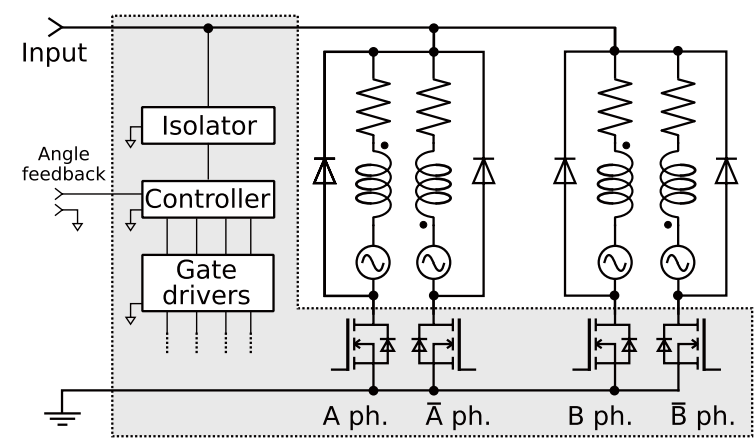

Fig. 5 Schematic of Routing Circuit for Motor Drive (RCMD) [9].

determines $p_{\mathrm{d}}$ at every clock time $t=k T(k=0,1, \cdots)$. The interval $T$ corresponds to the length of each power packet. The detailed algorithm for one cycle is as follows:

1. Obtain present angle position $\theta$ and its corresponding phase $p$ from the feedback signal. Note that $p \in$ $\{\mathrm{A}, \mathrm{B}, \overline{\mathrm{A}}, \overline{\mathrm{B}}\}$ is defined as the phase to be excited for holding the angle $\theta$.

2. Calculate the difference of target and present angles $D:=\theta_{\mathrm{d}}-\theta$, and then determine the target rotation direction as
a. clockwise, if $D>\theta_{\text {step }} / 2$,
b. counter-clockwise, if $D<-\theta_{\text {step }} / 2$,
c. holding present position, otherwise,

where $\theta_{\text {step }}$ is a step angle of the motor.

3. Determine the target phase $p_{\mathrm{d}}$ by appropriate shifting or holding of $p$ according to the result of Step 2.

The close-loop scheme enables angle control even in the presence of a disturbance thanks to the angle feedback signal in Step 1. In open-loop control, Step 1 is replaced by an estimation of $\theta$ and $p$ by the history of excited phases under the assumption that there occurs no unexpected angle error.

The control scheme above is applied to a distributed subsystem, which is a part of a larger dispatching network. Figure 4 shows the schematic of the subsystem. The subsystem consists of a stepper motor with an encoder and a routing circuit for motor drive (RCMD) [9]. RCMD, shown in 


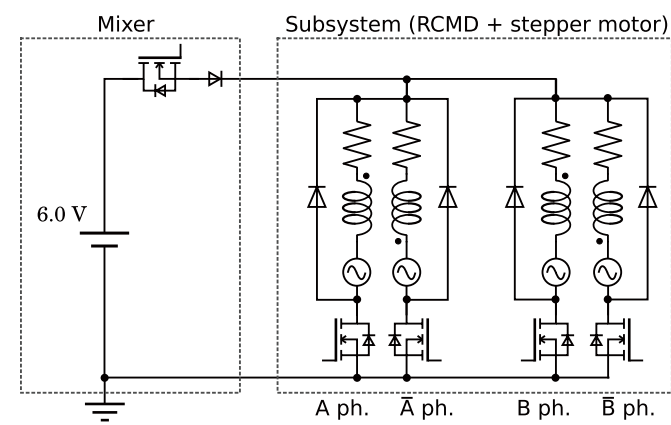

Fig. 6 Dispatching circuit used in experiment.

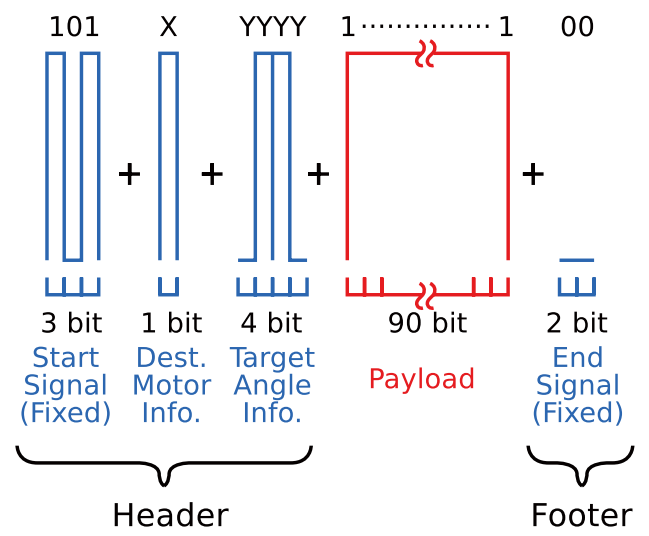

Fig. 7 Bit assignment of power packet. "1" and "0" represent "HIGH" and "LOW" in voltage, respectively.

Fig. 5, dispatches the payload of input power packets to motor phases by controlling the switches corresponding to the individual phases. Each power packet transferred to the subsystem contains the signal of the target angle $\theta_{\mathrm{d}}$. We assume the signal is determined and attached as a tag at the mixer, i.e. on the power source side. Every time the subsystem receives a power packet, the controller for RCMD determines the target phase according to the scheme explained above. Then RCMD dispatches the payload to the target phase. In this way, the angle control is decentralized; a part of control signals is generated on the load side.

\section{Motor Control Experiment}

\subsection{Experimental Setup}

Figure 6 depicts the dispatching circuit used in experiments. It consists of a voltage source of $6.0 \mathrm{~V}$, a mixer and a subsystem including a RCMD and a stepper motor (PKP214U06A-R2EL-L; step angle $\theta_{\text {step }}=1.8^{\circ}$ ). In this letter, router networks between the mixer and the subsystem are omitted without loss of generality; previous experimental study [5] already verified the power packet transfer via networked routers.

The configuration and bit assignment of power packet are shown in Fig. 7. The target motor signal of 1 bit in the header ( $X$ in Fig. 7 ) determines which motor to be fed by

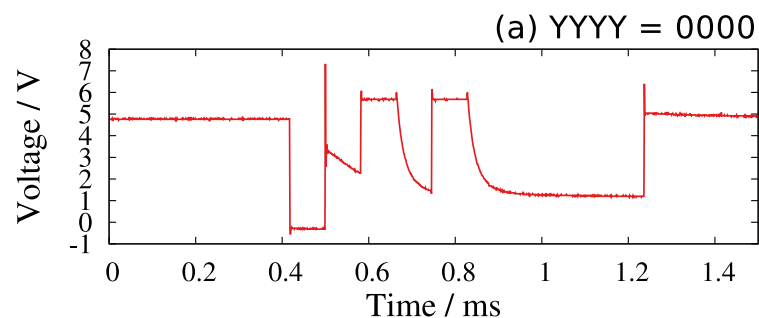

(b) $Y Y Y Y=0100$

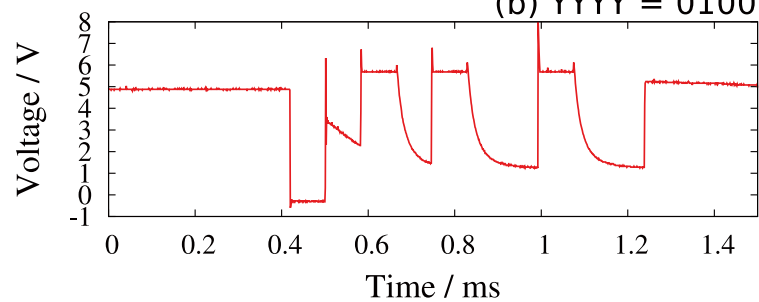

Fig. 8 Enlarged view of voltage waveforms of information tag, (a) YYYY $=0000,(b)$ YYYY $=0100$. A switching of RCMD occurred at the end of the previous packet (approximately at $0.5 \mathrm{~ms}$ ), which was followed by the header of the next packet (starting approximately from $0.6 \mathrm{~ms}$ ).

the payload. In the setup of this letter, we drive a single motor; therefore, $X$ is fixed at " 0 " for all the packets. The following 4 bit (YYYY in Fig. 7) determines the target angle signal as $(\mathrm{YYYY})_{10} \times 9^{\circ}$, where $(\cdot)_{10}$ indicates the conversion from binary to decimal. The length of 1 bit is set as $10 \times$ $2^{13} \mathrm{~ns}$ (approximately $80 \mu$ s); i.e. the control interval $T$ in the previous section is set as approximately $8 \mathrm{~ms}$.

The feasibility of the close-loop angle control is verified in experiments in the following two cases. In case 1, the target angle changes as $0^{\circ} \rightarrow 36^{\circ} \rightarrow 0^{\circ}$. The motor angle is controlled to follow the change of its target. In case 2, an angle disturbance caused by an impulsive force on counterclockwise direction is given to the motor while it is controlled to hold its present angle. We examine the improved response of the close-loop system to an unexpected disturbance by comparing the result with one using an open-loop scheme. As mentioned in the previous section, the openloop scheme uses the estimated angle, not the measured one, for angle control.

\subsection{Result of Case 1: with Changing Target Angle}

We first examine the information transfer via tags of power packets. Figure 8 shows an enlarged view of information tags in voltage waveforms measured at the input of RCMD. Figure 8 (a) is measured when the target angle is $0^{\circ}$, and (b) when $36^{\circ}$. In Fig. 8(a), the header indicates that the target angle is $0^{\circ}(\mathrm{YYYY}=0000)$. Similarly, in Fig. 8(b), the header indicates that the target angle is $36^{\circ}(Y Y Y Y=0100)$. At $0.5 \mathrm{~ms}$ in Figs. 8(a) and (b), a voltage spike occurred. This is because of the hard switching (from on to off) after RCMD recognized the footer, i.e. the end of the packet. In the setup of this letter, it was confirmed that the spike did not affect the information transfer.

Now, we confirm the achievement of angle control. Figure 9 (a) shows the measured angle trajectory. Mea- 


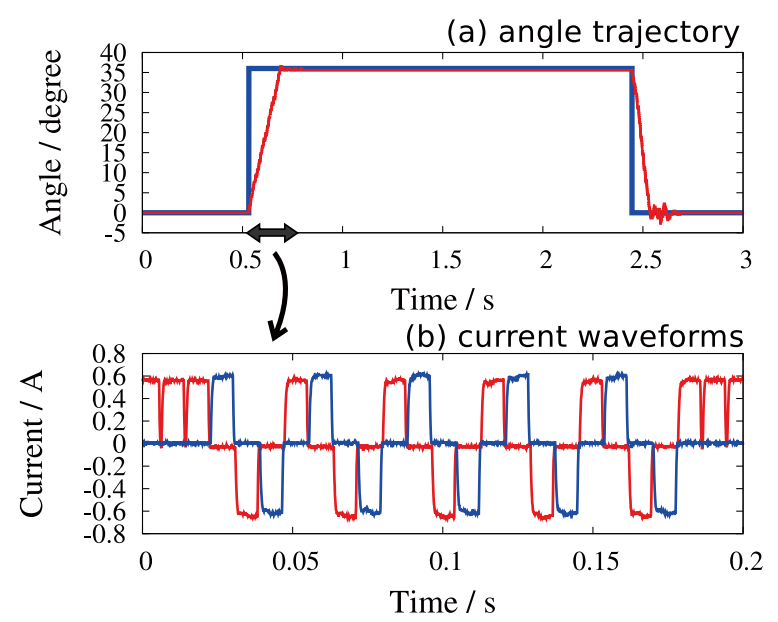

Fig. 9 Result of case 1: (a) measured angle trajectory (red) and its target (blue), (b) measured current waveforms (red and blue lines represent $i_{\mathrm{A}}-i_{\overline{\mathrm{A}}}$ and $i_{\mathrm{B}}-i_{\overline{\mathrm{B}}}$, respectively).

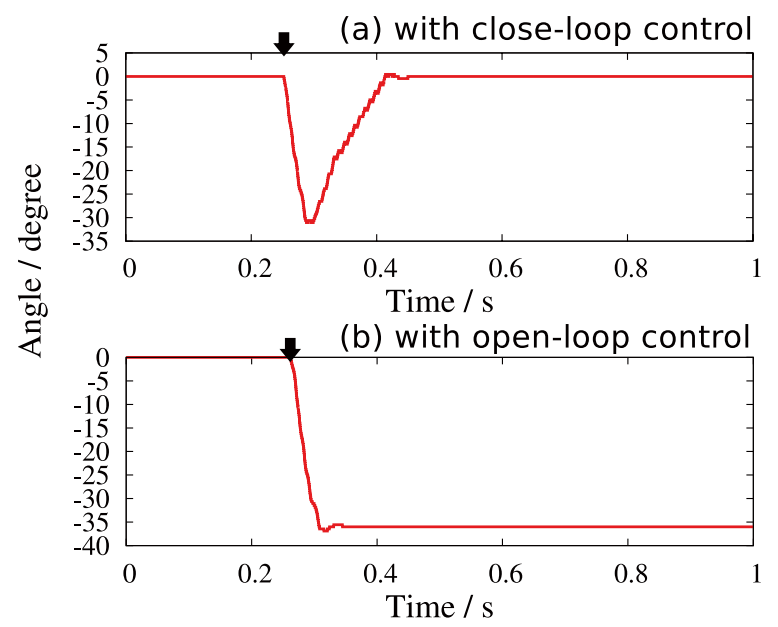

Fig. 10 Result of case 2: (a) measured angle with close-loop control, (b) measured angle with open-loop control. A disturbance was given at the time pointed by arrows.

sured trajectory followed the change of target angle $\left(0^{\circ} \rightarrow\right.$ $36^{\circ} \rightarrow 0^{\circ}$ ). Figure $9(\mathrm{~b})$ shows the measured current flowing the motor phases during the period indicated by the doubleheaded arrow in Fig. 9(a). Each waveform in the figure depicts the difference of current of complementary pair, e.g. $i_{\mathrm{A}}-i_{\overline{\mathrm{A}}}$; therefore, the positive (negative) value represents the excitation of $A(\overline{\mathrm{A}})$. Current pulses were given to the phases in the order corresponding to the clockwise rotation, "A $\rightarrow \mathrm{B} \rightarrow \overline{\mathrm{A}} \rightarrow \overline{\mathrm{B}} \rightarrow \cdots$," until the angle reached the target. From the results, the subsystem was confirmed to be able to control the destination phase appropriately using target and present angle signal.

\subsection{Result of Case 2: with Disturbance}

Now let us confirm the angle trajectories when a disturbance is given from outside the system. Figure 10(a) shows the angle trajectory controlled with the close-loop scheme. Af- ter the disturbance, the motor angle was controlled to get back to the target $0^{\circ}$ again. On the contrary, in Fig. 10(b) showing the result with the open-loop scheme, the angle did not get back to the target after the disturbance. Because the open-loop scheme has no means to sense the disturbance, it kept feeding to the same phase. The results indicate the proposed close-loop scheme achieves the angle control even in the presence of an unexpected disturbance.

\section{Conclusions}

In this letter, we verified that the proposed close-loop system achieved angle control of a stepper motor by integrated transfer of power and information, i.e. by power packets. The successful angle control was confirmed even in the presence of a disturbance. The results indicate that power packets can feed the power and information required for motion control. In addition, the decentralized implementation of the motion control scheme suggests the possibility of an application to a dispatching network consisting of many sources and loads.

\section{Acknowledgements}

This work was partially supported by Cross-ministerial Strategic Innovation Promotion Program from New Energy and Industrial Technology Development Organization and by the Super Cluster Program from Japan Science and Technology Agency.

\section{References}

[1] S. Seok, A. Wang, M.Y.M. Chuah, D.J. Hyun, J. Lee, D.M. Otten, J.H. Lang, and S. Kim, "Design principles for energy-efficient legged locomotion and implementation on the MIT cheetah robot," IEEE/ASME Trans. Mechatronics, vol.20, no.3, pp.1117-1129, June 2015.

[2] S.F. Tie and C.W. Tan, "A review of energy sources and energy management system in electric vehicles," Renew. Sustainable Energy Rev., vol.20, pp.82-102, April 2013.

[3] B. Sarlioglu and C.T. Morris, "More electric aircraft: Review, challenges, and opportunities for commercial transport aircraft," IEEE Trans. Transport. Electrific., vol.1, no.1, pp.54-64, June 2015.

[4] T. Takuno, M. Koyama, and T. Hikihara, "In-home power distribution systems by circuit switching and power packet dispatching," Proc. 1st IEEE Int. Conf. Smart Grid Commun., pp.427-430, Gaithersburg, MD, USA, Oct. 2010.

[5] R. Takahashi, K. Tashiro, and T. Hikihara, "Router for power packet distribution network: Design and experimental verification," IEEE Trans. Smart Grid, vol.6, no.2, pp.618-626, March 2015.

[6] S. Mochiyama, R. Takahashi, and T. Hikihara, "Trajectory control of manipulator fed by power packets," Int. J. Circ. Theor. Appl., Oct. 2016 (Published Online).

[7] T.R. Fredriksen, "New developments and applications of the closedloop stepping motor," J. Spacecraft and Rockets, vol.4, no.9, pp.11591165, Sept. 1967.

[8] T. Kenjo and A. Sugawara, Stepping Motors and Their Microprocessor Controls, 2nd ed., Oxford University Press, NY, 1994.

[9] N. Fujii, R. Takahashi, and T. Hikihara, "Application of power packet dispatching system to stepping motor driving system," Tech. on Electron. Devices and Semicond. Power Converter, IEE Japan, pp.53-58, Oct. 2014 (in Japanese). 